\title{
High dose rate brachytherapy for the local control of endobronchial carcinoma following external irradiation
}

\author{
Paul Hernandez, * Ashvini Gursahaney, Ted Roman, Kevin Schwartzman,
} David Donath, Manuel G Cosio, Robert D Levy

\begin{abstract}
Background - External irradiation is an established palliative treatment for patients with inoperable lung cancer. However, persistent or recurrent symptoms due to local disease are common following external irradiation. The impact of high dose rate (HDR) brachytherapy in the palliative management of patients with local sequelae of residual or recurrent endobronchial lung carcinoma following external irradiation was investigated.

Methods - A prospective cohort of 29 patients (19 men, mean age 65 years) underwent HDR brachytherapy for inoperable lung cancer. All patients had completed external irradiation at least one month before entry into the study (mean (SD) dose 4400 (1481) cGy, completed 12.9 $(21 \cdot 3)$ months previously). Patients underwent outpatient bronchoscopic placement of 1-3 HDR brachytherapy catheters for delivery of 750-1000 cGy of intraluminal irradiation every two weeks on 1-3 occasions. Prospective evaluation before and four weeks after completion of HDR brachytherapy included assessment of indices of level of function, symptoms, extent of atelectasis (chest radiography), and bronchoscopic determination of degree of endobronchial obstruction.
\end{abstract}

Results - One hundred and eighteen catheters were placed in 81 treatments. Eleven of the 26 patients who underwent repeat bronchoscopy showed a reduction in the degree of endobronchial obstruction; five of 18 patients had radiographic improvement in the extent of atelectasis. Positive response rates ranged from $25 \%$ for signs and symptoms related to pneumonitis to $69 \%$ for haemoptysis. Performance status improved in $24 \%$ of patients. Two patients died before completion of the study protocol. Short term complications included one episode of non-fatal, massive haemoptysis, five of minor haemoptysis, and one pneumothorax.

Conclusions - HDR brachytherapy may improve the degree of endobronchial obstruction, atelectasis, symptoms, and level of function with minimal short term complications in patients with recurrent or residual symptomatic disease following external irradiation.

(Thorax 1996;51:354-358)

Keywords: brachytherapy, lung cancer, external irradiation.

In North America there are approximately 175000 new cases of bronchogenic carcinoma diagnosed each year, ${ }^{12}$ of whom $60-80 \%$ have inoperable disease at presentation and another $10-20 \%$ are found to have extensive disease at the time of thoracotomy. ${ }^{3} \mathrm{Up}$ to $50 \%$ of patients eventually develop signs and symptoms referable to endobronchial disease including cough, haemoptysis, dyspnoea, and postobstructive pneumonitis. ${ }^{4}$

External irradiation is an established palliative treatment for patients with inoperable disease. $^{5-7}$ However, local symptomatic recurrence is common following external irradiation, occurring in approximately half of treated patients. ${ }^{8}$ Intolerance of normal surrounding tissues to irradiation may preclude further external beam therapy in these patients. High dose rate (HDR) remote afterloading endobronchial irradiation (brachytherapy) has recently become available as a therapeutic modality in this setting. Due to the rapid fall-off of the radiation delivered during brachytherapy, high doses can be delivered to target tissues while sparing normal surrounding tissues. ${ }^{9}$

Two recent papers have reported the results of brachytherapy in patients treated for symptomatic recurrent endobronchial tumour following previous maximal external beam irradiation. ${ }^{1011}$ Despite excellent symptomatic response rates in these studies, concern was raised regarding the high incidence of complications following brachytherapy, especially massive fatal haemoptysis. Whether this was related to the dosing schedule of brachytherapy or to coincident interventions - that is, laser debulking therapy - could not be explored due to the designs of the studies.

We report the results of a prospective evaluation of 29 patients treated at our institution with HDR brachytherapy for residual or re- 
current symptomatic endobronchial carcinoma following external irradiation. A substantial benefit of therapy was observed in some patients with a low incidence of serious adverse effects over the eight week study period.

\section{Methods}

STUDY POPULATION

Patients who met all of the following criteria were eligible for recruitment into the study: a history of histologically proven bronchogenic carcinoma; observable endoluminal tumour of the trachea, mainstem or lobar bronchi; recurrent or persistent symptoms (haemoptysis, cough, dyspnoea, or postobstructive pneumonitis) following previous maximal external irradiation as determined by the referring radiation oncologist; and consent to enter into the study. Exclusion criteria included patients with contraindications to flexible fibreoptic bronchoscopy or refusal by the patient to consent to the study protocol. The study protocol was approved by the institutional ethics review board.

From January 1992 to April 199429 consecutive patients (19 men) of mean age 65 years (range 36-80) who met the eligibility criteria were treated with HDR brachytherapy. Histological diagnoses included squamous cell carcinoma in 21 patients, adenocarcinoma in four, and small cell carcinoma in four. Among the patients with non-small cell carcinoma nine were stage IIIa, 13 stage IIIb, and three stage IV. ${ }^{12}$ Among the four patients with small cell carcinoma one was limited stage, while the others had extensive stage disease.

All patients had completed external irradiation at least one month before entry into the current study. The median (SD) interval from completion of external irradiation to the onset of brachytherapy was $12.9(21.3)$ months (range 1-25). Seven patients had completed external irradiation within two months of commencing brachytherapy treatment sessions. The mean dose of prior external irradiation was 4400 (1481) cGy. The average number of fractions per subject amounted to $20 \cdot 0(11.5)$, resulting in a mean fractional dose of 220 cGy. In addition to external irradiation, three patients had undergone endobronchial tumour debulking with Nd:YAG laser at least four weeks before brachytherapy. All four patients with small cell carcinoma had completed chemotherapy at least two months before commencing brachytherapy.

Patients demonstrated a broad range of performance status with a mean Eastern Cooperative Oncology Group (ECOG) score $^{13}$ at the time of initiation of the protocol of 1.9 $(0 \cdot 9)$. This scale (ranging from 0 to 4 ) is derived from a standardised questionnaire, with 4 representing the poorest performance status.

\section{BRACHYTHERAPY PROTOCOL}

Treatments were delivered on an outpatient basis. Patients underwent flexible fibreoptic bronchoscopy under local anaesthesia (eight puffs of $10 \%$ aerosolised xylocaine hydro- chloride by a metered dose unit and 3-5 $\mathrm{ml}$ of $2 \%$ liquid xylocaine). Patients were sedated with a benzodiazepine (midazolam 1-3 mg intravenously) and received a mucosal drying agent (atropine $0.4-0.6 \mathrm{mg}$ subcutaneously) before commencing the procedure.

The endobronchial tumour was identified and a hollow, closed ended polyurethane afterloading catheter (Nucletron, Leersum, Netherlands) was advanced through the suction channel of the bronchoscope beyond the site of the obstruction. If the lumen of the bronchus was completely occluded by tumour, the end of the catheter was placed adjacent to the obstructing mass. A guide wire was inserted through the catheter, allowing the bronchoscope to be removed while leaving the catheter in place. The guide wire was replaced with a dummy source with graduated radio-opaque markings $1 \mathrm{~cm}$ apart along its length. This procedure was repeated if catheters were placed in multiple locations. Multiple catheters were used for one of two reasons; firstly, to treat tumour identified at multiple endobronchial locations or, alternatively, to irradiate a greater volume of tissue as might be needed to treat disease located at a carina between two airways. ${ }^{14}$ The position of the catheter(s) was verified using fluoroscopy.

With knowledge of the airway anatomy, anteroposterior and lateral chest radiographs were used to determine a treatment plan with reference to the dummy source(s) placed during bronchoscopy. The high dose rate equipment utilised was the microSelectron-HDR (Nucletron, Leersum, Netherlands). This is a remote afterloading unit which uses a high activity (10 Ci) iridium-192 labelled source. The position and dwell-time of the iridium-192 source at the appropriate steps within the hollow catheter were calculated with a computer (MacIntosh Quadra 950) and dosimetry software developed at McGill University. ${ }^{15}$ Patients were treated over 10-20 minutes in an adjacent, shielded room during which time contact was maintained with personnel through a closed circuit television system.

Patients were treated every two weeks with a goal of three sessions. Twenty six of 29 patients completed the three planned treatments (mean $2.7(0.5)$ sessions/patient). A mean single fraction of 805 (49) cGy (range $750-1000$ ) at a treatment depth of $1 \mathrm{~cm}$ from the axis of the catheter was delivered at each session. The average treatment length used per catheter was $8.4(2.5) \mathrm{cm}$ (range $2-16 \mathrm{~cm}$ ). One catheter was placed on 45 occasions, two on 35 occasions, and three on one occasion (total of 118 catheters in 81 treatment sessions).

\section{MEASURED VARIABLES}

Patients underwent flexible fibreoptic bronchoscopy for brachytherapy treatment and assessment of degree of endobronchial obstruction on weeks 0,2 , and 4 . An additional bronchoscopy without treatment was performed on week 8 for assessment of local response to therapy. Still photographs were later made from video recordings of each bron- 
Table 1 Objective and symptomatic responses to brachytherapy

\begin{tabular}{llrrr}
\hline & $\begin{array}{l}\text { Subjects } \\
\text { evaluated }\end{array}$ & Improved & Worsened & Unchanged \\
\hline Endobronchial obstruction & 26 & $11(42 \%)$ & $2(8 \%)$ & $13(50 \%)$ \\
Atelectasis & 18 & $5(28 \%)$ & $5(28 \%)$ & $8(44 \%)$ \\
Haemoptysis & 16 & $11(69 \%)$ & $3(19 \%)$ & $2(12 \%)$ \\
Pneumonitis & 12 & $3(25 \%)$ & $7(59 \%)$ & $2(16 \%)$ \\
Dyspnoea & 29 & $7(24 \%)$ & $8(28 \%)$ & $14(48 \%)$ \\
Cough & 29 & $7(24 \%)$ & $6(21 \%)$ & $16(55 \%)$ \\
ECOG & 29 & $7(24 \%)$ & $10(34 \%)$ & $12(42 \%)$ \\
\hline
\end{tabular}

$\mathrm{ECOG}=$ Eastern Cooperative Oncology Group score

* The number of subjects evaluated represents the total number with the abnormality at the start of the study who underwent reassessment.

choscopic examination with comparable views of obstructed airways. Paired photographs from baseline and week 8 were assessed in random order by a blinded observer for degree of endobronchial obstruction. They were rated as improved (more than 25\% decrease in degree of endobronchial obstruction), worsened (more than $25 \%$ increase in obstruction), or unchanged.

The following were also evaluated at baseline (before brachytherapy) and at the visit by a
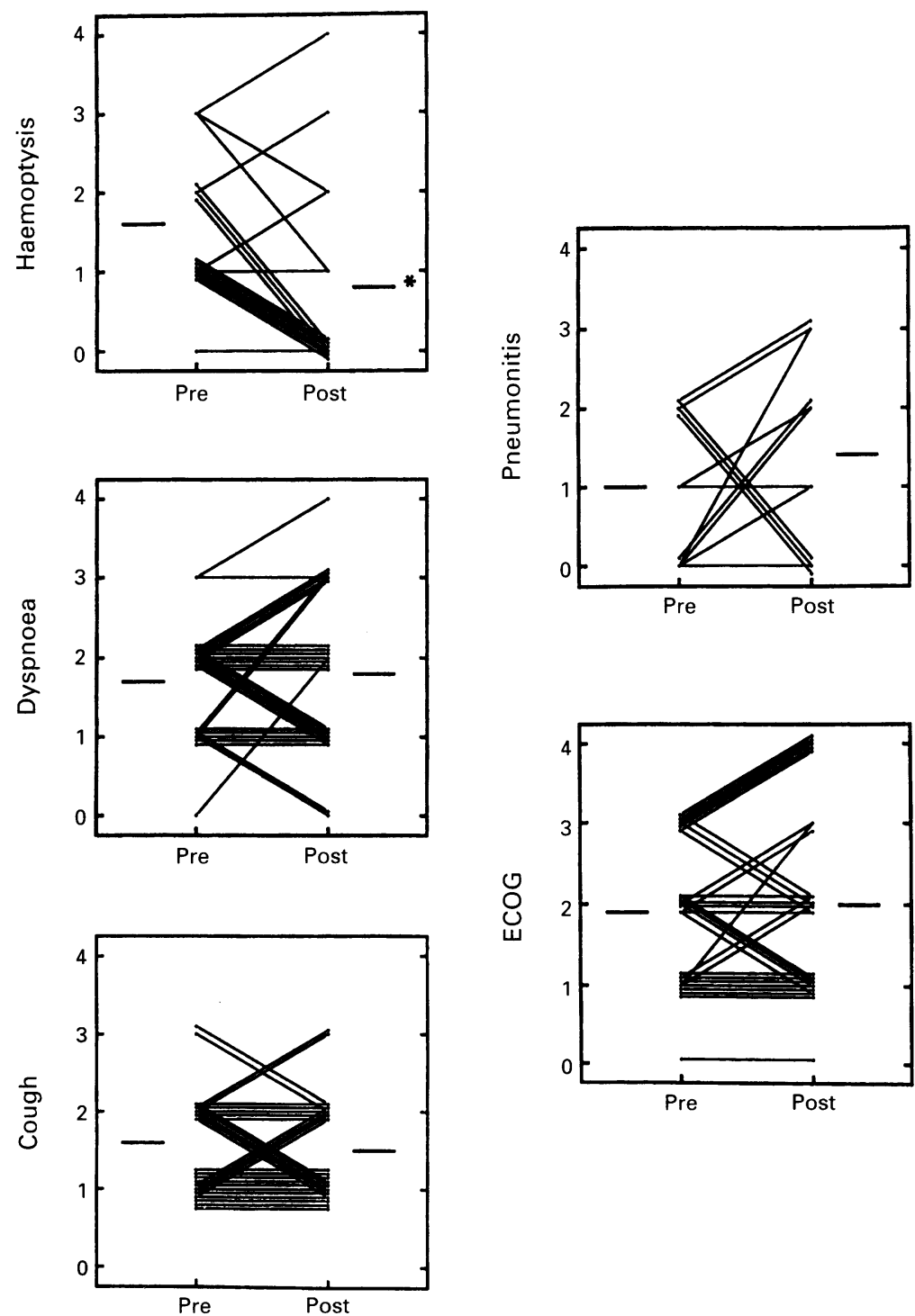

Scoring of symptoms, signs, and performance status (ECOG) before and after brachytherapy using numerical indices ${ }^{1316}$ ranging from 0 (best) to 4 (worst) for individual patients and group mean data (small horizontal bars). ${ }^{*} p<0 \cdot 01$, Wilcoxon signed rank test. member of the study team four weeks posttherapy:

(1) Posteroanterior and lateral chest radiographs were taken to assess the change in extent of atelectasis (present in 18 patients at the start of therapy).

(2) Scoring of signs and symptoms including haemoptysis, dyspnoea, cough, and postobstructive pneumonitis (fever, leucocytosis, and chest radiographic abnormality) using a numerical index. ${ }^{16}$

(3) Performance status was assessed using the ECOG scale. ${ }^{13}$

Data are expressed as mean (SD). The statistical difference between symptoms, signs, and performance status before and after brachytherapy was assessed using the Wilcoxon signed rank test, with $\mathrm{p}<0.05$ being considered statistically significant.

\section{Results}

Twenty nine patients entered the study protocol. Three did not complete therapy: one refused completion of treatment and two died (after undergoing one brachytherapy session each). Neither death was directly related to a brachytherapy treatment session but rather to progressive deterioration related to their underlying disease. All three patients had advanced stage disease and substantial constitutional symptoms at presentation, resulting in poor performance status scores (ECOG score of $3)$. None of these three patients underwent a second assessment for comparison with baseline so they were considered to have "worsened" for the purpose of evaluation of response rates of symptoms and performance status to brachytherapy.

\section{RESPONSE TO THERAPY}

Of the 29 patients entered into the study 26 underwent repeat bronchoscopies. Eleven patients $(42 \%)$ had more than $25 \%$ reduction in the degree of endobronchial obstruction, two worsened $(8 \%)$, while the remaining 13 patients $(50 \%)$ were unchanged at the completion of their treatment as compared with the initial assessment (table 1).

Eighteen patients had radiographic evidence of atelectasis before commencing therapy, and five of these showed improvement following brachytherapy. Four of the five patients who had evidence of improvement in the extent of atelectasis during the eight week study period also showed bronchoscopic improvement.

Table 2 Improvement rates for signs and symptoms in relation to change in endobronchial obstruction as assessed by bronchoscopy

\begin{tabular}{lll}
\hline & \multicolumn{2}{l}{ Bronchoscopic appearance } \\
\cline { 2 - 3 } & Improved & Worsened/unchanged \\
\hline Atelectasis & $4 / 9(44 \%)$ & $1 / 8(13 \%)$ \\
Haemoptysis & $5 / 7(71 \%)$ & $6 / 7(86 \%)$ \\
Pneumonitis & $3 / 6(50 \%)$ & $0 / 5(0 \%)$ \\
Cough & $6 / 11(55 \%)$ & $1 / 15(7 \%)$ \\
Dyspnoea & $4 / 11(36 \%)$ & $3 / 15(20 \%)$ \\
ECOG & $3 / 11(27 \%)$ & $4 / 15(27 \%)$ \\
\hline
\end{tabular}

ECOG $=$ Eastern Cooperative Oncology Group score. 
Response rates of symptoms and signs for individual patients as well as the group means following brachytherapy are shown in the figure. Improvement rates ranged from $24 \%$ in the case of cough to $69 \%$ for haemoptysis. There was a significant decrease in haemoptysis scores after brachytherapy $(\mathrm{p}<0 \cdot 01)$.

Rates of improvement were further assessed in relation to improvement or not in the degree of endobronchial obstruction (table 2). With the exception of haemoptysis, rates of improvement in signs and symptoms were considerably greater in those patients who showed a reduction in the degree of endobronchial obstruction than in those without bronchoscopic improvement.

Global performance status was assessed by ECOG scale. Performance status improved in $24 \%$ and remained stable in another $42 \%$ of patients. The performance status declined in the remaining $34 \%$ of patients. Patient survival, as determined from the time of entry into the study protocol, was $5.5(4.9)$ months (range 1-19).

\section{COMPLICATIONS OF THERAPY}

Minor haemoptysis ( $<100 \mathrm{ml}$ ) related to catheter placement occurred in six of 29 patients $(21 \%)$ or six of 81 treatment sessions $(7 \%)$. None of these patients required specific treatment or admission to hospital for the haemoptysis. There was one episode of non-fatal massive haemoptysis ( $>500 \mathrm{ml}$ of bloody expectoration in a 24 hour period) which occurred within two hours of a brachytherapy session. One patient experienced a pneumothorax related to catheter placement which did not require thoracostomy drainage. No patients had evidence of radiation bronchitis or stenosis.

\section{Discussion}

The recent development of a high dose rate remote afterloading system has led to the increased use of endobronchial brachytherapy in the treatment of inoperable bronchogenic carcinoma. Although external irradiation is an established palliative treatment for symptomatic inoperable lung cancer, the dose that can be delivered is limited due to radiation effects to the surrounding normal lung tissue and adjacent structures. Endobronchial brachytherapy circumvents this problem by providing a highly active irradiation source adjacent to the endobronchial disease with a rapid fall-off within a short distance.

The present study shows that a number of patients receiving brachytherapy for local sequelae of persistent or recurrent disease following previous maximal external irradiation have objective improvement in the degree of visible endobronchial obstruction and radiographic abnormalities. The response rates we observed are consistent with those previously described in the literature in those patients who received brachytherapy following treatment with external irradiation. ${ }^{101117}$ Furthermore, although progressive deterioration would be the expected course, a number of our patients showed improvement in symptoms of cough, haemoptysis, dyspnoea and those related to postobstructive pneumonitis. With the exception of haemoptysis, improvement rates for signs and symptoms were superior in those patients who had bronchoscopic evidence of diminished endobronchial disease. This suggests that symptomatic improvement was related to the HDR brachytherapy rather than to spontaneous recovery. A small number of patients even showed an improvement in global performance status. Brachytherapy therefore appears to be a useful palliative intervention in this group of patients with lung cancer for whom limited therapeutic options exist. However, conclusive proof of the benefit is currently lacking in the literature and could only be established by a controlled randomised trial comparing brachytherapy with either best supportive care (observation) or another treatment modality (laser therapy, photodynamic therapy, stenting, further external irradiation, etc).

Concern exists regarding the incidence rate of serious adverse effects of brachytherapy specifically major haemoptysis - in previously reported studies. ${ }^{17}$ Seagren et al treated 20 patients with HDR brachytherapy delivered in a single dose protocol of $1000 \mathrm{cGy}$ (four of whom also received endobronchial laser therapy) and reported a $25 \%$ incidence of fatal massive haemoptysis, ${ }^{10}$ and a $32 \%$ incidence of fatal haemoptysis was reported in the 38 patients treated by Bedwinek et al. ${ }^{11}$ All patients in the latter study received three fractions of 600 cGy HDR brachytherapy subsequent to endobronchial laser therapy. In an attempt to identify the factors that predispose to high complication rates, Nori et al reviewed the available literature and suggested that, although the evidence is inconclusive, the highest rates of fatal haemoptysis occurred in those patient series in which the highest dose per fraction of brachytherapy was used, especially when combined with laser debulking. ${ }^{17}$ The relative contribution of brachytherapy fractionation schedule to the incidence of serious complications is even less clear. In the current series we found that brachytherapy given in a protocol of three fractions of $800 \mathrm{cGy}$ at a treatment depth of $1 \mathrm{~cm}$ every two weeks is well tolerated in the short term by most patients with a low incidence of serious complications.

Radiation bronchitis and stenosis is a recently recognised complication of brachytherapy which can be graded by severity from a scale of I (mildest) to IV (most severe).$^{18}$ In the series reported by Speiser and Spratling ${ }^{16}$ the incidence of radiation bronchitis in those patients treated with $1000 \mathrm{cGy}$ at a depth of $1 \mathrm{~cm}$ for three sessions was $12 \%$. However, radiation bronchitis was not identified by those authors in the first six weeks following therapy. No radiation bronchitis or stenosis was observed in our series; however, endobronchial biopsies were not performed at follow up visits.

The short follow up (four weeks) and uncontrolled design of this study does not allow us to comment upon the durability of the benefits of treatment as well as the impact of 
brachytherapy on survival. Furthermore, these factors may account in part for the low incidence of massive haemoptysis and absence of radiation bronchitis or stenosis we observed compared with some previous reports. ${ }^{10111618-21}$

Three patients in the current series did not complete the intended treatment protocol. Two patients died from causes thought to be unrelated to the treatment sessions; the third refused further intervention for his lung cancer. All three patients had advanced disease and substantial constitutional symptoms at the time of their entry into the study. Although other patients with advanced stage disease and poor performance status received considerable benefit from HDR brachytherapy, it is possible that this subgroup of patients is at risk to suffer more from the discomfort of the intervention than to derive palliation of their symptoms. A larger cohort would be required to determine if factors such as initial performance status of the patient play a part in the outcome of treatment with HDR brachytherapy.

The authors wish to thank the Radiation Oncology technician and nurses, as well as the technicians of the Department of Inhalation Therapy of the Royal Victoria Hospital.

1 Canadian cancer statistics 1992. Toronto, Canada: National Cancer Institute of Canada. April 1992.

2 Cancer facts and figures. Atlanta: American Cancer Society, 1992

3 Fraser RG, Pare JAP, Pare PD, Fraser RS, Genereux GP. Diagnosis of diseases of the chest. Vol 2, 3rd edn. Philadelphia: WB Saunders, 1989: 1464

4 Moylan D. Overview of endobronchial brachytherapy and review of the literature. Presented at AER-ALTS II, New Orleans, 1987

5 Slawson RG, Scott RM. Radiation therapy in bronchogenic carcinoma. Radiology 1979;132:175-6.
6 Chetty KG, Moran EM, Sassoon CSH, Viavathana T, Light $R$. Effect of radiation therapy on bronchial obstruction due to bronchogenic carcinoma. Chest 1989;95:582-4.

7 Majid DA, Lee SK, Khushalani S, Seydel H. The response of atelectasis from lung cancer to radiation therapy. Int $\mathcal{F}$ Radiat Oncol Biol Phys 1986;12:231 -2.

8 Speiser BL. Remote afterloading brachytherapy for local control of endobronchial carcinoma. Int $\mathcal{f}$ Radiat (Oncol Biol Phy's 1992;25:579-87.

9 Hernandez P, Donath D. High dose rate endobronchial brachytherapy in the treatment of bronchogenic carcinoma. Chest 1993;104:989-91.

10 Seagren Sl, Harrell JH, Horn RA. High dose rate intraluminal irradiation in recurrent endobronchial carcinoma. Chest 1985;88:810-4

11 Bedwinek J, Petty A, Bruton C, Sofield J, Lee L. The use of high dose rate endobronchial brachytherapy to palliate symptomatic endobronchial recurrence of previously irradiated bronchogenic carcinoma. Int 7 Radiat Oncol Biol Phys 1992;22:22-30.

12 Mountain CF. A new international staging system for lung cancer. Chest 1986;89:225S.

13 Zubrod CG, Schneiderman M, Frei E. Appraisal of methods for the study of chemotherapy of cancer in man: comparative therapeutic trial of nitrogen mustard and triparative therapeutic trial of nitrogen mustard and tri-
ethylene thio phosphoramide. $f$ Chron Dis 1960;11:7-33.

14 Speiser B. Endobronchial brachytherapy: an overview. In Mould RF, ed. Brachytherapy 2. Leersum: Nucletron, 1989:463-8.

$15 \mathrm{Pla}$ C. Fast intracavitary and interstitial treatment planning. In Mould RF, ed. Brachytherapy 2. Leersum: Nucletron, 1989:109-20.

16 Speiser BL, Spratling L. Remote afterloading brachytherapy for the local control of endobronchial carcinoma. Int $f$ Radiat Oncol Biol Phys 1993;25:579-87.

17 Nori D, Allison R, Kaplan B, Samala E, Osian A, Karbowitz $\mathrm{S}$. High dose-rate intraluminal irradiation in bronchogenic carcinoma. Chest 1993;104:1006-11.

18 Macha HN, Koch K, Stadler M, Schumacher W, Krumhaar D. New technique for treating occlusive and stenosing tumors of the trachea and main bronchi: endobronchial irradiation by high dose iridium-192 combined with laser irradiation by high dose iridium-192

19 Mehta M, Shahabi S, Jarjour N, Steinmetz M, Kubsad S. Effect of endobronchial radiation therapy on malignant Effect of endobronchial radiation therapy on
bronchial obstruction. Chest 1990;97:662-5.

20 Speiser BL, Spratling L. Radiation bronchitis and stenosis secondary to high dose rate endobronchial irradiation. Int f Radiat Oncol Biol Phys 1993:25:589-597.

21 Burt PA, O'Driscoll BR, Notley HM, Barber PV, Stout R. Intraluminal irradiation for the palliation of lung cancer with high dose rate Micro-Selectron. Thorax 1990;45: 765-8. 